\title{
Repositorios institucionales digitales de acceso abierto: una mirada socio- tecnológica \\ Estudio de caso del repositorio de la Universidad Nacional de Entre Ríos - Argentina
}

\section{Gonzalo Andrés}

Universidad Nacional de Entre Ríos. Facultad de Ciencias de la Educación. Instituto de Estudios Sociales (UNER-Conicet).

Paraná, Argentina | gonzalo.andres@uner.edu.ar / https://orcid.org/oooo-0003-4993-6080

\section{Carina Cortassa Amadio}

Universidad Nacional de Entre Ríos. Facultad de Ciencias de la Educación. Paraná, Argentina | carina.cortassa@uner.edu.ar / https://orcid.org/oooo-0003-1585-022X

\section{Andrés Wursten}

Universidad Nacional de Entre Ríos. Facultad de Ciencias de la Educación. Paraná, Argentina | andres.wursten@uner.edu.ar / https://orcid.org/oooo-0002-0837-1039

\section{Juan Ignacio Legaria}

Universidad Nacional de Entre Ríos. Facultad de Ciencias de la Educación. Paraná, Argentina l juanlegariacdu7@gmail.com / https://orcid.org/oooo-0001-5320-4742

\section{Resumen}

En este artículo se describe y analiza el desarrollo socio-tecnológico del Repositorio Institucional (RI) de la Universidad Nacional de Entre Ríos (UNER, Argentina) durante el período 2015-2019. El estudio forma parte de un proyecto de investigación más amplio, enfocado en una variedad de estrategias y prácticas institucionales destinadas a promover la comunicación científica y el acceso abierto al conocimiento. Desde la perspectiva de los Estudios Sociales de la Tecnología, se concibe a los RI como dispositivos complejos, en cuyo diseño e implementación se articulan soportes y artefactos técnicos, decisiones institucionales, normativas, hábitos culturales, prácticas de uso, afinidades, intereses y disputas entre grupos sociales. Mediante un abordaje metodológico cualitativo -basado en fuentes documentales, entrevistas en profundidad y grupos focales de discusión- se logró identificar un conjunto de potencialidades y dificultades presentes en la estrategia difusionista adoptada por la UNER en el desarrollo de su repositorio digital. Si bien ello permitió cumplir con los requerimientos legales y tecnológicos externos, el análisis muestra que en el proceso se soslayaron las necesidades, prácticas y hábitos de los diversos tipos de agentes internos involucrados -dimensiones clave para la apropiación grupal y la sostenibilidad socio-técnica de un RI.

\section{Palabras clave}

Repositorios Institucionales Acceso Abierto Enfoque Socio-Técnico Sostenibilidad

Universidad Nacional de Entre Ríos 
Open access institutional digital repositories: a socio-technical approach. A case study of the repository of the Universidad Nacional de Entre Ríos - Argentina

\section{Keywords \\ Institutional repositories Open Access Socio-technical approach
Sustainability Universidad Nacional de Entre Ríos}

Abstract

A description and analysis of the socio-technical development of the Universidad Nacional de Entre Ríos (UNER, Argentina) Institutional Repository (IR) is presented. The study is part of a wider research project, focused on a variety of institutional strategies and practices devoted to promote science communication and the open access to knowledge. Relying on the Social Studies of Technology broad perspective, IRs are conceived as complex devices, whose design and implementation show tight links among technical means and artifacts, institutional decisions, norms, cultural habits, use practices, group affinities, interests and disputes. Qualitative data gathered through documentary sources, in-depth interviews and focused groups discussions, lead to identify a set of possibilities and difficulties involved in the diffusion strategy adopted by the University in the development of its digital repository. While allowing the institution to comply with applicable legal and technological requirements, that course of action also overlooked the internal relevant actors' particular needs, practices and habits - all of them key dimensions for IR's group appropriation and socio-technical sustainability.

Artículo recibido: 20-01-2020. Aceptado: 05-05-2020

\section{Introducción}

La problemática en torno a la circulación del conocimiento y los procesos de mediatización de la ciencia contemporánea no es reciente, pero ha adquirido una relevancia central en el contexto de la llamada Sociedad de la Información (Nieto-Galán, 2011; Burke, 2017). La dimensión comunicacional ya no se percibe como un factor exógeno ni como una actividad destinada a difundir a posteriori los avances de una disciplina, sino como una característica estructural y estructurante de las instituciones y las prácticas científicas contemporáneas (Polino y Castelfranchi, 2012). A ello se suma la creciente expansión de las tecnologías informáticas y digitales, que están transformando sustancialmente los procesos de producción, circulación y acceso a la información y el conocimiento. La digitalización de contenidos, la aparición de sitios web especializados y los motores de búsqueda, así como la conformación de enciclopedias y plataformas colaborativas, trajeron consigo la puesta en crisis del modelo tradicional de publicación y acceso al conocimiento (Bartling y Friesike, 2014).

Desde comienzos del siglo XXI se viene consolidando a nivel mundial un movimiento que promueve el Acceso Abierto (AA) al conocimiento científico-técnico a partir del aprovechamiento de las potencialidades de las Tecnologías de la información y la Comunicación (TIC). La Declaración de Budapest (BOAI, 2002) y la Declaración de Berlín (2003) constituyeron los hitos fundantes de este movimiento. Desde entonces, se entiende al acceso abierto como la posibilidad de acceder mediante internet al conocimiento y el patrimonio cultural aprobado por la comunidad científica. Ello implica que cualquier persona pueda buscar, leer, descargar, copiar y distribuir artículos, con la única limitación de respetar los derechos patrimoniales (copyright) de los autores y el derecho a ser adecuadamente reconocidos y citados. 
Para alcanzar ese propósito se postularon dos posibles vías: la "dorada" y la "verde". La primera propone que todas las revistas científicas permitan el acceso libre y gratuito a sus contenidos; la segunda apela a la actitud proactiva de los autores a difundir su producción, a través del auto-archivo de sus trabajos en repositorios institucionales o temáticos. Desde entonces, la proliferación de revistas académicas de AA -la "vía dorada"-se fue intensificando, de manera coherente y convergente con el creciente interés de las comunidades y grupos de investigación por dar visibilidad a su producción. Esta cuestión remite, por un lado, a los cambios que generó internet en las prácticas de acceso a la información; por otro, a la crisis de legitimidad de las revistas comerciales tradicionales (Luchilo, 2019). Asimismo, con el correr de los años se expandió también la instrumentación de repositorios -la "vía verde"-, que se focalizan en la creación de colecciones digitales de la producción científico-tecnológica que permitan su posterior búsqueda y recuperación, aunque no impliquen necesariamente una reestructuración total del sistema de edición (Bueno de la Fuente y Hernández Péres, 2011; Barrueco Cruz et al., 2014).

Transcurrida una década desde el planteamiento original se elaboró una serie de recomendaciones tendientes a consolidar la iniciativa (BOAI, 2012). Esos mandatos apuntaron especialmente a la necesidad de contar con legislaciones nacionales específicas y con la formulación e implementación de estrategias institucionales concretas para la comunicación y circulación del conocimiento. Entre ellas, se destaca la sugerencia de que cada organismo de investigación y/o educación superior disponga de su propio RI para publicar los artículos, tesis y disertaciones producidos en el transcurso de sus actividades sustantivas ${ }^{1}$. En este marco, los Repositorios Institucionales (RI) constituyen en la actualidad uno de los dispositivos socio-tecnológicos más valiosos para potenciar el carácter "público" de las ciencias, especialmente en dos sentidos: promover su comunicación y evitar su privatización ${ }^{2}$.

En este contexto internacional, en la República Argentina se conformó en 2010 el Sistema Nacional de Repositorios Digitales (SNRD), que nuclea los repositorios de las instituciones que reciben financiamiento público y establece los criterios tecnológicos y bibliotecológicos básicos para que puedan ser incluidos en el mismo. Vale decir que para entonces ya existían iniciativas pioneras en el país, como por ejemplo el Servicio de Difusión de la Creación Intelectual de la Universidad Nacional de La Plata (SEDICIUNLP, 2003), la Biblioteca Digital de la Universidad Nacional del Litoral (2006) o el Repositorio Hipermedial de la Universidad Nacional de Rosario (2009). ${ }^{3}$

Posteriormente, en 2013 se sancionó la Ley $\mathrm{N}^{\circ} 26.899$ que establece que los organismos e instituciones públicas integrados en el sistema nacional de ciencia y tecnología deben desarrollar RI digitales, propios o compartidos. El propósito es que allí se publique en AA la producción científico-tecnológica (artículos de revistas, trabajos técnico-científicos, tesis académicas, entre otros) financiada total o parcialmente con fondos públicos. La ley establece, asimismo, que el incumplimiento de sus disposiciones expone a las entidades a posibles sanciones, consistentes en la no-elegibilidad en las convocatorias a recursos de apoyo a la investigación y desarrollo de agencias estatales (Art. $8^{\circ}$ Ley $26.899 / 2013$ ) 4 .

En ese contexto, el propósito de este artículo es analizar el desarrollo durante el período 2015-2019 del repositorio de la Universidad Nacional de Entre Ríos (RIUNER) (http://ri.uner.edu.ar). Se presenta un estudio de caso en profundidad acerca de las políticas, estrategias y prácticas mediante las cuales esta institución de educación superior afronta el compromiso de implicarse con los procesos de circulación y apropiación social del conocimientos.

La investigación, de carácter cualitativo, abordó la problemática comunicacional en dos escenarios: el de las interacciones inter-pares y el de la comunicación pública de las ciencias. Si bien es cierto que los RI tienen la potencialidad de promover la
1. Al respecto, se cuenta con el registro internacional de políticas de acceso abierto (Registry of Open Access Repository Material Archiving Policies), el cual realiza una documentación permanente del crecimiento de mandatos de acceso abierto en distintos países.<http:// roarmap.eprints.org>

2. Nuestro enfoque de los RI de AA asume como puntos de partida que: a) la ciencia no es talen ausencia de comunicación como afirma Price (1965: 58); y b) que la libre disposición que permiten los RI del conocimiento garantiza la "publicidad" de los bienes científicos, eludiendo su transformación en mercancías y la tendencia a la privatización de sus beneficios que deriva de ello.

3. Para un detalle sobre los repositorios pioneros en la Argentina, veáse el trabajo de C. De Volder (2008)

4. La aprobación de esta normativa obligó a todos los organismos del sistema científico-tecnológico a avanzar en la configuración socio-técnica de sus respectivos Repositorios Institucionales. Para un análisis detallado de este proceso ver el trabajo de Fushimi (2018).

5. Proyecto de I+D “Circulación, acceso y apropiación social del conocimiento científico y tecnológico: acciones y prácticas en la Universidad Nacional de Entre Ríos", PID $N^{\circ} 3166$, realizado entre 2017-2019 con financiación de la Secretaría de Ciencia y Técnica de dicha universidad. Más detalles sobre el caso objeto de estudio se aportan en la sección 2. Metodología. 
accesibilidad al conocimiento en ambos sentidos, en la actualidad sus usos concretos se vinculan estrechamente con las prácticas, intereses y necesidades de las comunidades científicas y académicas. Por esa razón, este segmento de nuestro trabajo se enfocó en los agentes institucionales, en particular en el modo en que sus actitudes y percepciones constituyen un fuerte condicionante de las prácticas relativas al desarrollo e implementación del repositorio.

\section{Los RI como dispositivos socio-tecnológicos}

El enfoque conceptual adoptado para este análisis se inscribe en la tradición de los estudios sociales sobre la tecnología inaugurada por el Programa SCOT (Social Construction of Technology). En particular, en la discusión que entablan con aspectos centrales de las perspectivas difusionistas sobre el cambio y la innovación tecnológica en distintos ámbitos, consolidadas durante la década de 1960.

Brevemente, estas suponen que tras la creación y desarrollo de artefactos o conocimiento tecnológicos se requiere de una vigorosa campaña dedicada a su difusión entre los potenciales beneficiarios o usuarios. En esa línea, Rogers (1962) postuló que las variables que intervienen en los procesos de transferencia serían: a) las características propias del objeto técnico o conocimiento tecnológico creado; b) su efectiva difusión por diversos canales; c) la cantidad de tiempo "prudencial" para que los sujetos la incorporen; y d) las características del sistema social que recibe la innovación. Desde ese enfoque se distinguen dos momentos en la innovación tecnológica: en primer lugar, una innovación sería una idea, una práctica o un objeto que es percibido como nuevo por un sujeto o una unidad de adopción; en segundo lugar, la difusión es un proceso mediante el cual la innovación es comunicada en el tiempo y difundida por determinados canales, entre los miembros de un entorno social. Este modelo fue exitoso durante las décadas de 1960 y 1970, pero posteriormente fue criticado en varios aspectos: a nivel operativo (por sus falencias en algunas experiencias), a nivel ideológico (por su mirada "neutral" y "bondadosa" de la tecnología), y a nivel epistémico (por otorgar demasiado peso a la comunicación para explicar el cambio social) (Beltrán, 1976; Cimadevilla, 2004; Martin y Osberg, 2007).

Durante las últimas décadas del siglo pasado, en el campo de los estudios sociales de la tecnología se fue consolidando una mirada diferente sobre la creación, desarrollo y usos sociales de una tecnología. Entre otros referentes, la denominada "perspectiva socio-técnica" surgió de trabajos pioneros como el de Pinch y Bijker (2008 [1987]), en los cuales se plantearon una serie de argumentos originales relativos a la contextualidad de los efectos sociales del uso de los objetos técnicos y a la existencia de grupos sociales relevantes con intereses en pugna que intervienen en la construcción de la utilidad de un artefacto. Por lo tanto, toda tecnología es resultado de un complejo entramado socio-técnico, donde intervienen tanto los soportes y artefactos técnicos como las decisiones institucionales, las normativas y exigencias, los hábitos culturales, las prácticas de uso extendidas y las afinidades, intereses y disputas entre grupos sociales. Así, el desarrollo y el "funcionamiento" (en términos de Pinch y Bijker, ob.cit) de una tecnología no se define a priori, sino que comporta una contingencia construida social y tecnológicamente. En consecuencia, en los procesos de construcción de una tecnología intervienen dinámicas complejas en las que se combinan regulaciones sociales y legislaciones, hábitos culturales, actividades con fines de lucro, criterios éticos y estéticos, conocimientos científicos, formas de poder y regímenes de relación social.

Con el tiempo, el campo de los estudios sociales de la tecnología se fue enriqueciendo con aportes provenientes de la teoría de sistemas, la teoría del actor-red y el 
constructivismo social ${ }^{6}$. Todas esas miradas comparten el propósito de evitar determinismos sociales o tecnológicos derivados de abordajes monocausales. Es decir, se apunta a explicar las relaciones socio-técnicas evitando distinciones a priori entre lo "tecnológico", lo "social", lo "político", lo "económico" y lo "científico" (Thomas, Becerra y Bidinost, 2019). Por tanto, un enfoque socio-técnico de la mediatización no evalúa "impactos" de una tecnología sobre las prácticas sociales, sino que estudia cómo se interrelación las lógicas de las tecnologías de comunicación con las lógicas de las otras instituciones sociales (Verón, 2015).

En cuanto a nuestro objeto de estudio, Guédon (2009) afirma que los RI son un buen caso para ejemplificar las características de los "artefactos socio-técnicos", ya que allí confluyen redes sociales y saber experto, por lo que para su análisis es pertinente la adopción de la mirada constructivista que propone el Programa SCOT. Por tanto, bajo esas premisas, nuestro análisis del RI-UNER apunta a desentrañar la densa trama de artefactos, sujetos, organizaciones, conocimientos, reglamentaciones, prácticas y discursos que intervienen en su construcción.

Al respecto vale mencionar que, si bien recientemente están surgiendo publicaciones en ese sentido, aún no son frecuentes los estudios que aborden la creación y utilización de repositorios desde una mirada constructivista como la expuesta. Sin embargo, entre la literatura especializada es posible mencionar diferentes trabajos que delinearon la pertinencia de este enfoque socio-técnico. Por ejemplo, se destacan los trabajos de Rieger (2008) y Guédon (2009) como los primeros en promover este tipo de enfoque para el análisis de los repositorios. Posteriormente, se encuentra el análisis de un repositorio latinoamericano de Babini et al. (2010); las propuestas de líneas estratégicas de acción planteadas por Severin (2013); el modelo metodológico y tecnológico propuesto por San Martín, Guarnieri y Bongiovani (2014); las evaluaciones y recomendaciones técnicas y socio-institucionales de Callicott, Scherer y Wesolek, (2016) sobre un conjunto de repositorios mexicanos; y la exhaustiva investigación realizada por Fushimi (2018) sobre la construcción de repositorios en Argentina durante el período 2004-2015.

Siguiendo esa línea, nuestra investigación se centró en las interrelaciones entre las prácticas de publicación, los hábitos culturales y las decisiones socio-institucionales que influyeron -como se verá- en la escasa utilización y sostenibilidad del RI-UNER. Se considera que, en última instancia, estos son los factores que en mayor medida contribuyen a la sostenibilidad (o no) de una plataforma de publicación en AA de artículos académicos. De hecho, la literatura producida a nivel internacional durante las últimas dos décadas muestra que su éxito depende de un involucramiento de todos los grupos sociales en la configuración del dispositivo, así como de un cambio en las rutinas de búsqueda de información y publicación de artículos por parte de los agentes institucionales (Bueno de la Fuente y Hernández Péres, 2011; San Martín et al., 2013; Kennison y Norberg, 2014).

Participar colaborativamente en la generación y publicación de conocimiento, así como en la sostenibilidad de un dispositivo socio-tecnológico, implica mucho más que acceder a contenidos o interactuar con artefactos. Así, pues, se propone pensar la sostenibilidad de las prácticas y los artefactos a partir de las dinámicas sociales, culturales y pedagógicas (en todo proceso media siempre alguna forma de aprendizaje) de la institución en la que se desarrolla. De modo que se entiende que la sostenibilidad de un RI se manifiesta en un proceso de convalidación social, cierta perdurabilidad en el tiempo y un crecimiento de la red socio-técnica inicial. Esto significa, entre otras cosas, que la sostenibilidad requiere ir más allá de las condiciones iniciales favorables para la construcción del dispositivo. Con todo, la adopción de un enfoque socio-técnico habilita a pensar el ciclo de
6. Para una revisión detallada de la evolución de los estudios sobre los procesos de innovación y creación tecnológica, véase el artículo de Tabares Quiroz y Correa Vélez (2014). 
vida de una tecnología (y el entramado socio-tecnológico que lo sustenta) más allá de las miradas de "difusión" o "transferencia", así como la evaluación de su uso en términos de "impacto".

\section{Metodología}

Los resultados presentados en este artículo corresponden a un estudio de caso en profundidad, realizado entre 2017-2019, cuyo propósito fue describir y analizar de qué modo se encararon las demandas emergentes relativas al desarrollo socio-tecnológico de un RI de AA desde la Universidad Nacional de Entre Ríos.

La UNER es una institución pública de educación superior argentina creada en 1973, que cuenta actualmente con nueve Unidades Académicas distribuidas en seis localidades de la provincia de Entre Ríos: Paraná (Facultades de Ciencias de la Educación, Trabajo Social y Ciencias Económicas); Oro Verde (Facultades de Ingeniería y Ciencias Agropecuarias); Concordia (Facultades de Ciencias de la Administración y Ciencias de la Alimentación); Gualeguaychú (Facultad de Bromatología); Villaguay (sub-sede de la Facultad de Ciencias de la Salud); Concepción del Uruguay (Facultad de Ciencias de la Salud y Rectorado). En el organigrama institucional, el RI-UNER depende de la Secretaría Académica del Rectorado.

El análisis partió de los siguientes interrogantes:

1. ¿Qué medidas adoptó la Universidad en el proceso de creación y desarrollo de un repositorio institucional para cumplimentar lo establecido por la Ley 26.899 sancionada en 2013.

2. ¿De qué manera se están afrontando los cambios que implica -tanto a nivel político- institucional como en el plano de las representaciones, rutinas y hábitos de los grupos sociales involucrados- la implementación de un dispositivo de esta índole?

3. ¿Fueron contempladas las actitudes y percepciones de los grupos sociales involucrados en el diseño del RI, a los fines de lograr una sostenibilidad socio-técnica en el tiempo?

4. ¿En qué medida las estrategias y decisiones institucionales adoptadas para la creación y desarrollo del RI-UNER incidieron en las prácticas y hábitos de los agentes involucrados?

El diseño metodológico fue de carácter cualitativo, exploratorio-descriptivo y sincrónico, basado en fuentes primarias (entrevistas y grupos focales) y secundarias (documentos y reglamentaciones). Particularmente, las entrevistas y grupos focales se realizaron durante el periodo comprendido entre mayo de 2018 a junio de 2019. Se efectuaron veintidós entrevistas en profundidad a informantes clave -autoridades rectorales y de las unidades académicas, responsable de la coordinación del RI y bibliotecarios/as de Facultades-; también se concretaron cuatro grupos focales de discusión con investigadores/as de distintas disciplinas.

Si bien en el transcurso de la investigación se abordaron también aspectos eminentemente tecnológicos, en los resultados que se presentan a continuación se enfatizan dos niveles de análisis: 1) el nivel macro, de las políticas, estrategias y acciones institucionales relativas al desarrollo del RI-UNER; y 2) el nivel micro, de las percepciones y actitudes de diversos grupos de agentes respecto de sus prácticas y responsabilidades relativas a la cuestión. Esto, por un lado, es consecuente con el enfoque teórico adoptado; por otro lado, es producto de la propia investigación: como las evidencias indican, la escasa utilización actual del RI-UNER 
y los problemas identificados para su sostenibilidad socio-técnica a futuro tienen que ver con factores socio-institucionales antes que tecnológicos.

\section{Resultados}

\subsection{Política institucional: diseño y desarrollo del RI-UNER}

La UNER inició la creación y desarrollo de su RI en 2015, con la firma de una Carta Acuerdo de colaboración con la UNLP por un año y prorrogable por otro más; es decir, el plan de trabajo allí previsto se extendía hasta octubre de 2017.El acuerdo se basó fundamentalmente en dos motivos. Por un lado, se consideró que no existía en la UNER ni infraestructura técnica ni personal experto para la implementación del repositorio. Por otra parte, como ya fue mencionado, el SEDICI-UNLP fue pionero en el tema en Argentina y es el mejor reputado de nuestro país desde el 20127. El objetivo principal de la Carta Acuerdo consistía en que la Dirección del SEDICI colaborase con la creación y puesta en marcha del RI-UNER mediante la instalación y configuración del software y la capacitación de personal especializado. Según el convenio, las actividades incluirían el dictado de cursos, formación de docentesinvestigadores, tareas de difusión científica, acciones de capacitación/consultoría, transferencia de tecnología y proyectos de investigación conjuntos.

En ese marco se definieron programas de trabajo anuales para avanzar en la puesta en práctica. La ejecución, seguimiento y evaluación de las actividades quedó a cargo de un grupo conformado por representantes de ambas instituciones, bajo la dirección de una Coordinadora -especialista en Bibliotecología- dispuesta por la UNER.

Para desarrollar las tareas de configuración del RI-UNER se establecieron tres objetivos: a) definir políticas de metadatos, formatos y organización del repositorio; b) poner en funcionamiento la plataforma DSpace (el software adoptado por la mayoría de las universidades argentinas); y c) capacitar al personal informático y administrativo. Para ello se organizó un plan de trabajo en cuatro etapas (Tabla 1), al término del cual la Universidad tendría que haber dispuesto de un dispositivo acorde a las recomendaciones del SNRD.

También se realizó la consiguiente capacitación al personal afectado a las tareas -tanto en Rectorado como en las Facultades-, aprobándose asimismo designaciones específicas para que cada unidad académica se ocupe de la carga de archivos y edición de metadatos. Paralelamente a la concreción de esas etapas, en diciembre de 2016 se completaron los trámites de adhesión al SNRD. El siguiente paso en este organismo consistiría en la aprobación de las políticas institucionales para la publicación en AA -requisito que, como se señala en la sección siguiente, todavía no ha sido cumplimentado.

Como se observa, la UNER logró resolver la implementación y configuración tecnológica del repositorio mediante la contratación de servicios externos:

"El diseño fue todo realizado por la gente del SEDICI. Se copió... O sea, ellos implementaron acá la misma producción que hicieron para el diseño del repo de su universidad. (...) Lo que nosotros vamos a implementar son las colecciones propias que va a haber en cada una de las unidades, según las necesidades de cada una de las facultades" (CO-RIUNER, la cursiva es propia).

8. Los testimonios de los informantes se identifican mediante la referencia a la función de quien los expresa: CO-RIUNER: Coordinadora del Repositorio; SEC: Secretario/a de Universidad o de Facultad; BIB: Bibliotecario/a. INV: Investigador/a. Las intervenciones se integran en el texto de dos maneras: entre comillas, en el contexto de la idea desarrollada en un párrafo, o en párrafo aparte -distinguible por sangrado y tamaño de la tipografía- cuando se considera oportuno destacarla. Se procuró no modificar sustancialmente los usos del lenguaje propios de la oralidad salvo cuando fuera necesario por cuestiones de coherencia textual y legibilidad. 


\begin{tabular}{|l|l|}
\hline 1. Etapa de Diseño & $\begin{array}{l}\text { Definición de políticas sobre metadatos, formatos, } \\
\text { autoarchivo, organización del repositorio y servicios. Tareas } \\
\text { de diseño gráfico y maquetación. Se realizó una clase-taller } \\
\text { con el personal afectado al repositorio en cada unidad } \\
\text { académica. }\end{array}$ \\
\hline $\begin{array}{l}\text { 2. Etapa de } \\
\text { Implementación }\end{array}$ & $\begin{array}{l}\text { Customización del software DSpace (v. 6) a los } \\
\text { requerimientos de metadatos, permisos, organización y } \\
\text { circuitos de carga establecidos en la etapa 1; instalación } \\
\text { y configuración en servidores del Rectorado. La decisión } \\
\text { de adoptar la tecnología utilizada por el SEDICl-UNLP } \\
\text { excluyó la evaluación técnica de otras opciones de software } \\
\text { existentes, tampoco se consultó a personal interno } \\
\text { (informáticos y bibliotecarios) ni se consideró la alternativa } \\
\text { de desarrollar un software propio9. }\end{array}$ \\
\cline { 2 - 3 } desarrollo del RI-UNER & $\begin{array}{l}\text { 3. Etapa de Pruebas } \\
\text { Puesta en funcionamiento de un prototipo del repositorio } \\
\text { en un servidor web, actividades de prueba y carga inicial de } \\
\text { materiales. Se efectuaron capacitaciones para los recursos } \\
\text { humanos afectados al RI-UNER, bajo un esquema de clases } \\
\text { teóricas y tutorías prácticas para informáticos y } \\
\text { administradores. }\end{array}$ \\
\hline Customización & $\begin{array}{l}\text { Se concretó hacia fines de 2017. Incluyó la corrección de } \\
\text { problemas, la puesta a punta de servicios de software y } \\
\text { hardware, la configuración de metadatos para la indexación } \\
\text { en Google Scholar y generación de estadísticas del acceso a } \\
\text { comunidades, colecciones, ítems y descarga de archivos. }\end{array}$ \\
\hline
\end{tabular}

9. Como se verá en breve, el hecho de no promover suficientemente la participación de la comunidad local en la discusión sobre las opciones socio-tecnológicas, ni contemplarse la posibilidad de que recursos humanos propios realicen una adaptación y/o actualización de alguna de las plataformas disponibles, tuvo fuertes repercusiones en la percepción y actitudes de los agentes involucrados, comprometiendo así la sostenibilidad del dispositivo.

\subsection{Política institucional: limitaciones en la configuración del RI-UNER}

Finalizado el período comprendido por la Carta Acuerdo UNER-UNLP, el asesoramiento del SEDICI se discontinuó y el proceso de diseño y desarrollo del RI pasó a depender exclusivamente de decisiones y acciones intra-institucionales. Durante el 2018 se inició una etapa de elaboración, discusión y evaluación en el Consejo Superior de la Universidad sobre las políticas del repositorio digital institucional. Se consideró que, tras haberse resuelto los requerimientos técnicos, era necesario establecer normativas para su uso y funcionamiento (tal como lo exige el SNRD). No obstante, hacia fines de 2019 esas políticas no habían sido aprobadas.

De lo anterior se desprende que, concluida la consultoría externa, la puesta en marcha del RI-UNER no registró avances significativos. De hecho, el propio Consejo Superior reconoció oportunamente que "su desarrollo es incipiente y no resulta suficiente, por lo que deberán desarrollarse políticas específicas para disponer de bibliografía fuente en formato digital y accesible" (Resol. C.S. 15/18). Por su parte, en el orden práctico, desde el Rectorado se planteó en más de una ocasión a los responsables de las bibliotecas de cada Facultad la posibilidad de incorporar equipamientos para la digitalización del material en soporte papel, pero esa iniciativa nunca fue concretada. No en vano, luego de un cambio de gobierno en la Universidad, una de las nuevas autoridades afirmaba a mediados de 2018 que el propósito de la gestión entrante sobre el RI era que:

“Lo mínimo es tenerlo. Y empezar a tener los procedimientos para montar las tesis de grado o de posgrado. (...) Hemos estado charlando. O sea: [tenemos que] reconocerlo, institucionalizarlo, ver hacia dónde tenemos que apuntar, ver lo lejos que estamos..." (SEC., la cursiva es propia). 
Hasta el momento, la publicación en AA de la producción científica de la UNER se encuentra paralizada. Eso sitúa a la Universidad en condiciones de incumplimiento de la legislación vigente a nivel nacional y la expone, como se indicó previamente, a ser pasible de las sanciones establecidas en la Ley 26.899 de 2013.

\subsection{Actitudes y percepciones sobre el AA}

En el marco de la investigación se consultó además a informantes clave -autoridades de Facultades, investigadores/as, responsables de bibliotecas- sobre sus actitudes y percepciones sobre el AA y el desarrollo del RI-UNER. Aunque también se plantearon objetivos diferentes en cada caso: entre las autoridades, se trató de determinar su nivel de conocimiento acerca del tema; entre las y los docentes-investigadores y responsables de bibliotecas interesaban sobre todo sus actitudes y percepciones sobre el desarrollo institucional del dispositivo.

Este último grupo de agentes manifestó en su totalidad conocer las demandas y exigencias de la legislación nacional y estuvo de acuerdo con su implementación. En términos generales, bibliotecarios y bibliotecarias demostraron un marcado interés en que la Universidad cuente con repositorio propio, así como también valoraron positivamente la difusión en AA de la producción científico-tecnológica local. No obstante, también plantearon dudas y reticencias sobre el proceso de diseño y desarrollo del RI-UNER, por motivos de diversa índole: en particular, sobre los modos de gestión organizacional, la adopción de decisiones tecnológicas inconsultas, la escasa difusión del proyecto y el visible retraso en definiciones legales y normativas. A continuación se detallan algunos de los aspectos principalmente objetados.

\section{Los modos de gestión organizacional}

A lo largo del proceso de configuración socio-tecnológica del RI-UNER, las autoridades de la universidad buscaron incorporar tecnologías y saberes externos para luego intentar un posterior desarrollo propio. Esta decisión generó críticas por parte de los encargados de bibliotecas, que son en definitiva quienes tendrán que llevar a cabo la consolidación del dispositivo en el mediano plazo.

Vale mencionar que durante el periodo de discusión y sanción de la Ley Nacional $\mathrm{N}^{\circ} 26.899$-entre los años 2012 y 2013-, estos agentes siguieron de cerca el debate legislativo, organizaron charlas con referentes sobre el tema y se reunieron con sus autoridades para visibilizar la situación. Incluso en algunas Facultades se proyectó la posibilidad de diseñar repositorios propios. Pero esas iniciativas fueron discontinuadas luego de que en 2015 el Rectorado anunciara el proyecto de un repositorio único y centralizado.

Como ya se expresó, dicho proceso se inició ese año con la firma de un Carta Acuerdo con el SEDICI/UNLP. Esto ocurrió a pesar de que en la propia Universidad había recursos humanos especializados que mostraron interés e inquietud en el diseño e implementación de un repositorio. La concreción de las actividades estipuladas en esa Carta Acuerdo también contribuyó a cimentar dificultades y diferencias. La asesoría del SEDICI se materializó en la instalación y puesta a punto del servicio informático, y en el dictado de charlas de capacitación a los involucrados localmente en el tema. Esas capacitaciones constituyeron, sobre todo, instancias de transferencia de saberes tecnológicos y de experiencia de gestión en la carga de información, pero no se focalizaron en las situaciones, necesidades e intereses particulares de cada una de las unidades académicas de la UNER. Como sostiene una entrevistada: 
“Desde mi punto de vista, está muy verde el tema. Hubo una contratación a la gente de la UNLP, que tienen el repositorio armado. La bajada de ellos fue la contratación puntual fue la parte informática, sistema y demás. Pero en realidad si nos ponemos a pensar en un proyecto, hay políticas primero, hay que debatir qué es lo que vamos a cargar y qué no. Todo eso es lo más engorroso, ponernos de acuerdo, y después ver qué software vamos a usar y demás. Creo que hubo un mal manejo ahí, que por un lado me da la sensación que es para mostrar y en concreto hoy en día no tenemos nada" (BIB-s, la cursiva es propia).

Por otro lado, la decisión de contratar un personal externo para coordinar el RI fue tomada de manera "sorpresiva" por los encargados de las bibliotecas, quienes percibieron en ello desconocimiento y desinterés acerca de sus propios planteamientos e iniciativas. Si bien no se cuestiona su idoneidad, sí se objeta que

“fue un nombre que surgió de una asesoría técnica que hubo en las bibliotecas (...) no fue un nombre que surgió desde el seno de la Universidad. (...) En verdad nos sorprendió que sea una persona ajena a la institución la que esté coordinando el repositorio. Es algo medio... raro" (BIB-3).

En efecto, la estrategia de las autoridades de la universidad se basó en una lógica de transferencia, adoptándose una expertise de terceros para el desarrollo propio. Esta decisión profundizó disidencias entre las y los encargados de bibliotecas. Esa desazón se evidenció en los testimonios de los informantes:

"Hay mucha gente capacitada en la UNER y no se la valora. ¿Siempre hay que ir a buscar afuera teniendo tanta gente con tanto valor en nuestra universidad?" (BIB-5).

"Cuando hicimos la última acreditación, en 2014, nosotros propusimos un plan de mejora sobre un repositorio institucional de la Facultad, no de la Universidad (...) Íbamos a empezar a trabajar y en 2015 sale la Universidad con la propuesta. Entonces tuvimos una reunión y yo les digo: 'nosotros tenemos todo armado, están los archivos listos para cargar, todo'. Al final decidimos acoplarnos con el repositorio de la universidad y dejar el proyecto nuestro que ya teníamos todo estructurado. Bueno... la Universidad se ha demorado como un año y pico para empezar a armar todo y al final se contrató a la gente del SEDICI de La Plata..." (SECyT-3).

\section{Escaso conocimiento del proyecto}

Las y los responsables de las bibliotecas también cuestionaron la nula discusión y difusión sobre los lineamientos legales, tecnológicos e institucionales del RI en la universidad. Esto se materializó, de hecho, en el bajo nivel de conocimiento que reportaron los agentes que participaron de las entrevistas y grupos focales de nuestra investigación. Por su parte, entre las autoridades de las Facultades esa percepción es sumamente variable. Hay quienes admiten que "no están empapados" en el tema; quienes sostienen que su conocimiento es superficial, "muy por arriba", "poco y nada", "es una cuenta pendiente"; o quienes dicen estar al tanto de la existencia de la legislación nacional pero no sobre las acciones de la universidad.

Fueron pocos los informantes que afirmaron haberse involucrado en el proceso, ya sea a partir de las iniciativas que se venían proyectando en sus propias Facultades o por haber participado en la gestación o uso de repositorios en otros contextos institucionales. Solamente tres de los entrevistados (sobre un total de 22) reflejaron 
en sus testimonios un conocimiento de cierta profundidad sobre la temática: pueden, por caso, aludir a la controversia sobre la propiedad intelectual de las producciones finales; a las dificultades inherentes a la disposición y manejo de metadatos; a la relación entre la problemática del AA con las revistas científicas y con los mecanismos de evaluación académica; a los alcances de la ley en cuanto al tipo de contenidos a incluir en los repositorios, etc.

Entre los y las investigadoras, la sensación de ajenidad resulta bastante similar a la demostrada por las autoridades de las Facultades. Si bien los términos "repositorio institucional" o "acceso abierto" se asocian con cuestiones varias, en general se percibe su relación con: a) la necesidad de disponibilizar la producción científica financiada con recursos públicos; $\mathrm{y} b$ ) centralizar los esfuerzos individuales orientados a ponerla en AA -por ejemplo, en plataformas como ResearchGate, Academia.edu o similaresreclamando nuevamente el liderazgo firme desde la institución.

"Lo que tengo entendido es que toda la info la podés dejar en un repositorio para que pueda ser utilizada por otros investigadores que quieran trabajar con esa información. Siempre hay un resguardo de la info de base, pero vos podés trabajar. Se usa mucho en EEUU (...) En ese sentido estoy a favor de compartir la información, esta tiene que ser pública, más en un lugar público-en el que nos paga el Estado para hacerlo. Sea Universidad, CONICET, INTA, donde sea, la información tiene que estar disponible, obviamente con resguardo en las cuestiones de autoría" (GF-3).

"Calcularía que es como en otros países: que cualquier cosa que esté financiada por el Estado argentino tiene que ser accesible a cualquier ciudadano del Estado argentino de manera abierta (...) En tanto y en cuanto la Facultad no lo centralice, o la Universidad no lo centralice, no se va a visibilizar, porque no es lo mismo entrar a través del repositorio de la UNER que entrando en ResearchGate. Eso nos fragmenta como instituto o centro de producción del conocimiento: uno de acá, otro de allá y uno del otro lado, en lugar de decir -como hacemos en CONICET"vamos a la página de este a bajar algo'” (GF-2).

Si bien entre los docentes-investigadores el conocimiento sobre el tema no es extendido, quienes están más interiorizados demandan su implementación en la UNER. Sin embargo, la información acerca de las acciones de la institución es muy escasa: "no sé demasiado" fue la expresión más reiterada en los grupos focales cuando se aludió al tema. En el mismo sentido, se reiteraron afirmaciones del tipo "no tengo idea", "nunca supe que se estuviera haciendo", "falta difusión, porque no estaba enterada", "yo tampoco conocía que la UNER estaba con eso", "nunca me consultaron".

Estas observaciones condicen, en parte, con las decisiones adoptadas por las autoridades. Desde Rectorado se dispuso que, en el proceso de desarrollo, la primera etapa estuviese destinada a la instalación del software necesario, la preparación de los servidores y la puesta en línea de subdominio en la web. Y se previó que un segundo momento del proyecto estaría dedicado a la difusión de la tecnología instalada y a la concientización de los docentes sobre la problemática. Así lo indicaba la Coordinadora del Repositorio hacia mediados de 2018:

"Nosotros ahora estamos trabajando en la difusión. Por ejemplo, en octubre transmitimos las charlas del mes del acceso abierto. Desde Rectorado se difunde mucho este tema. Es importante, por eso hay que insistir. Capaz habría que armar más difusión o más prensa desde las bibliotecas, comunicando qué es el acceso abierto" (CO-RIUNER) (la cursiva es propia).

En similar dirección se manifiesta un secretario de Facultad: 
“Falta hacer difusión de esto, falta... Precisamente estoy por armar un curso interno de motivación, adonde voy a explicar cómo funciona, para qué se hace, por dónde viene la cosa: 'dentro de dos años ustedes van a presentar un proyecto y van a tener que hacer un plan de gestión de datos, esto se arma así y así'. Tirarles 50 6 pautas: ‘miren muchachos la cosa viene por acá, yo les advierto’. (...) Hay que hacer toda una tarea de concientización, de charlar con los investigadores para que vean cómo se cargan los datos, qué datos necesitan, qué información hay que disponer..." (SECyT-3).

\section{Discusión}

A partir de los resultados expuestos, es posible afirmar que las políticas institucionales de la UNER tendieron a impulsar la configuración tecnológica del RI para cumplimentar la legislación vigente antes que a generar interés por parte de la comunidad científica en el tema. Para ello, incorporó en el organigrama institucional una Coordinación del RI durante el periodo 2015-2018, con una responsable a cargo, y destinó presupuesto específico, lo cual constituye un aspecto positivo que expresa el interés político que se le asigna y la voluntad de concretarlo, a pesar de todas las dilaciones ya expuestas.

Sin embargo, no se diagramó el desarrollo de un RI a partir del involucramiento de los grupos sociales implicados (bibliotecarios, informáticos, docentes-investigadores), ni se establecieron políticas de publicación en AA o manuales de procedimiento para la carga de la producción. Por el contrario, la UNER adoptó para el desarrollo de su RI una estrategia difusionista (de tipo top-down), que tuvo como propósito dar a conocer entre los agentes universitarios esta innovación de publicación y circulación de la producción científica. Sin embargo, a partir del análisis presentado, es posible afirmar que esta estrategia comunicacional no fue exitosa, en el sentido de que no logró usos y apropiaciones estables y expandidos del repositorio.

En lo concerniente a las actividades, también es preciso señalar que el proceso de implementación del RI-UNER se encuentra en un virtual stand-by, con todo lo que ello implica en cuanto al desaprovechamiento del impulso original y de las inversiones realizadas (en la consultoría de la UNLP, la compra de equipamiento y la capacitación de recursos humanos). Las acciones encaradas desde el Rectorado -en sentido top-down-fueron numerosas, pero hasta el momento revisten una falencia muy significativa que atenta contra la sostenibilidad socio-técnica del RI-UNER.

Como se observa, la disponibilidad de una tecnología en un marco socio-institucional no garantiza su integración en las prácticas cotidianas (San Martín et al., 2016). Incluso los propios promotores del AA a nivel internacional han planteado que la cantidad de depósitos en los repositorios no crece si no es fomentada la práctica de auto-archivo de textos por parte de las y los investigadores (BOAI, 2012).

Los usos estabilizados e institucionalizados de una tecnología comportan una construcción socio-tecnológica sostenida en el tiempo (Verón, 2015). Y, en este caso, de acuerdo con la información relevada, la construcción socio-tecnológica del RI-UNER no surgió de las necesidades de los docentes-investigadores ni del trabajo en conjunto de los bibliotecarios e informáticos, ni tampoco se los comprometió en su proceso y evolución. De allí el riesgo de ser considerado exclusivamente como una exigencia externa (una ley sancionada a nivel nacional) o un desarrollo externo (realizado por Rectorado con colaboración del personal de la UNLP). No en vano, finalizado el trabajo de SEDICI, la Universidad por sí misma no pudo realizar avances concretos: más allá de que el software DSpace esté en línea, luego de cinco años de políticas 
top-down aún no se han generado las condiciones institucionales y las prácticas culturales necesarias para la posible sostenibilidad socio-técnica y estabilidad en el tiempo del RI-UNER.

Resulta dificultoso que la comunidad científica local se sienta interpelada por el desarrollo del RI y/o por la publicación en AA: ante una política de hechos consumados, lo que cabe esperar es que “(...) primero van a pataleary después se van a tener que adaptar. Porque aparentemente lo que se viene es que en los formularios de los proyectos para financiamiento se van a pedir que se hagan los esquemas de datos que va a usar el proyecto, como lo va a difundir, como lo va a publicar, o sea que va a ser una parte del proyecto, todo sí o sín (SECyT-3, la cursiva es propia).

En síntesis, desde el Rectorado de la Universidad y sus dependencias se adoptó un rol preeminente en el desarrollo del repositorio, asumiendo el liderazgo de las acciones sin detenerse en motivar una implicación temprana de los agentes internos involucrados. Esa forma de gestión e implementación del proyecto contribuyó a generar reticencias y cuestionamientos. En esas condiciones, resulta poco factible lograr las transformaciones culturales que -a la par de las tecnológicas- se requieren para promover cambios reales en las formas de publicación, circulación y consumo de la producción científica. La UNER se abocó a la exigencia de ajustarse a los marcos normativos, avanzando para ello en la dimensión técnica del RI (diseño del sitio web), pero depositó escasa atención en la dimensión social intrínseca a todo dispositivo de esta naturaleza. Las dificultades actuales a las que se enfrenta su implementación se pueden comprender a partir del interés secundario -según se infiere de nuestros resultados- asignado para promover el involucramiento y participación efectiva de los diversos agentes institucionales en el proceso.

\section{Conclusión}

En un contexto internacional signado por una creciente mediatización de las instituciones y prácticas científicas, sumado a las legislaciones nacionales que promueven el AA a las publicaciones académicas, la puesta en marcha de un RI constituye un aporte concreto a la materialización del postulado ético-político de la democratización del conocimiento científico en las sociedades contemporáneas. En este artículo se presentó un análisis socio-técnico del desarrollo del RI de la UNER donde se evaluó -desde una perspectiva cualitativa- la estrategia institucional implementada por las autoridades, así como las actitudes y percepciones de las y los investigadores al respecto.

La estrategia adoptada por la universidad se basó en una lógica de transferencia (de tecnología y saberes) y de difusión (de una plataforma de publicación) que no fue suficiente para generar interés por parte de los grupos sociales involucrados. Durante la etapa de diseño y desarrollo se pusieron de manifiesto las afinidades, tensiones y diferencias entre los distintos agentes implicados. Esto se evidenció, por ejemplo, en el reclamo recurrente de los bibliotecarios para participar en la toma de decisiones tecnológicas y políticas, en las que no tuvieron intervención a pesar de ser ellos los encargados del mantenimiento, actualización y carga de materiales.

La adopción de un enfoque socio-técnico para realizar un estudio de estas características pone de manifiesto que la configuración de una tecnología no es determinada a priori, sino que se van definiendo en la propia dinámica del proceso de co-construcción. En este caso, se considera que las prácticas individuales o colectivas de uso de un repositorio universitario será el resultado de una reformulación de las tareas de investigación y docencia, que implique una resignificación de los hábitos, percepciones y saberes de los docentes-investigadores, así como su inclusión en 
proyectos institucionales de comunicación pública de las ciencias. Por lo tanto, independientemente de que exista una legislación nacional o que haya una plataforma en línea, resulta dificultosa la sostenibilidad socio-técnica del RI-UNER si la comunidad académica de la universidad lo entiende como una exigencia más, o no lo considera como una iniciativa que responda a una mejora de la práctica educativa y a la mayor difusión y circulación de su producción. 


\section{Q Referencias bibliográficas}

» Babini, Dominique, Jessica González, Fernando López y Flavia Medici. 2010. Construcción social de repositorios institucionales: el caso de un repositorio de América Latina y el Caribe. En Información, Cultura y Sociedad. No. 23, 63-90. <http://revistascientificas.filo.uba.ar/index.php/ICS/article/view/746> [Consulta: 17 enero 2020].

»Bartling, Sönke y Sascha Friesike, eds. 2014. Opening Science. The Evolving Guide on How the Internet is Changing Research, Collaboration and Scholarly Publishing. Berlín: Springer.

» Barrueco Cruz, José Manuel, Mercedes de Miguel Estévez, Cristina González Copeiro y Pilar Rico-Castro, eds. 2014. Guía para la evaluación de repositorios institucionales científico. Madrid: Fundación Española para la Ciencia y la Tecnología.

"Beltrán, Luis Ramiro. 1976. Alien premises, objects, and methods in Latin American communication research. En Communication Research. Vol. 3, no. 2, 107134. <https://doi.org/10.1177/009365027600300202>

"Berlín Declaration on Open Access to Knowledge in the Sciences and Humanities. 2003. $<$ https://openaccess.mpg.de/Berlin-Declaration> [Consulta: 17 enero 2020].

»BOAI. 2002. Budapest Open Access Initiative. Budapest: Open Society Institute. <http://www.budapestopenaccessinitiative.org/read> [Consulta: 17 enero 2020].

"BOAI. 2012. Ten years on from the Budapest Open Access Initiative: setting the default to open. Budapest: Open Society Institute. <https://www.budapestopenaccessinitiative.org/boai-10-recommendations> [Consulta: 17 enero 2020].

"Bueno de la Fuente, Gema y Tony Hernández Péres. 2011. Estrategias para el éxito de los repositorios institucionales de contenido educativo en las bibliotecas digitales universitarias. En BiD: textos universitaris de biblioteconomia i documentació. No. 26. <https://dx.doi.org/10.1344/105.000001727>

»Burke, Peter. 2017. ¿Qué es la historia del conocimiento? Buenos Aires: Siglo Veintiuno editores.

»Callicott, Burton, David Scherer y Andrew Wesolek. 2016. Making Institutional Repositories Work. Indiana: Purdue University Press.

»Cimadevilla, Gustavo. 2004. Dominios. Crítica a la razón intervencionista. Buenos Aires: Prometeo.

"De Volder, Carolina. 2008. Los repositorios de acceso abierto en Argentina: situación actual. En Información, Cultura y Sociedad. No. 19, 79-98. <http://revistascientificas.filo.uba.ar/index.php/ICS/article/view/833> [Consulta: 17 enero 2020].

"Fushimi, Marcela. 2018. Desarrollo de repositorios digitales institucionales en las universidades nacionales en Argentina, periodo 2004-2015. Tesis de Maestría. Bernal: Universidad Nacional de Quilmes. <http://ridaa.unq.edu.ar/handle/20.500.11807/887>

»Guédon, Jean-Claude. 2009. Es un repositorio, es un depósito, es un archivo. Open Access, colecciones digitales y valor. En ARBOR Ciencia, Pensamiento y Cultura. Vol. 85, no. 737, 581-595. <http://doi.org/10.3989/arbor.2009.i737.315> 
»Kennison, Rebecca y Lisa Norberg. 2014. A Scalable and Sustainable Approach to Open Access Publishing and Archiving for Humanities and Social Sciences. New York: KIN Consultants.

»Ley № 26.899. 2013. Repositorios digitales institucionales de acceso abierto. Argentina. Sistema Nacional de Ciencia, Tecnología e Innovación. <http://servicios.infoleg.gob.ar/infolegInternet/anexos/220000-224999/223459/norma. htm> [Consulta: 17 enero 2020].

»Luchilo, Lucas. 2019. Revistas científicas: oligopolio y acceso abierto. En Ciencia, Tecnología y Sociedad. Vol. 14, no. 40, 41-79. <http://www.revistacts.net/files/Volumen_14_Numero_40/o2Luchilo.pdf> [Consulta: 17 enero 2020].

"Martin, Roger y Sally Osberg. 2007. Social Entrepreneurship: the case for definition. En Stanford Social Innovation Review. Vol. 5, no. 2, 29-39. <https://ssir.org/ articles/entry/social_entrepreneurship_the_case_for_definition> [Consulta: 17 enero 2020].

»Nieto-Galán, Agustí. 2011. Los públicos de la ciencia. Expertos y profanos a través de la historia. Madrid: Marcial Pons.

»Pinch, Trevor y Wiebe Bijker. 2008 [1987]. La construcción social de hechos y artefactos: 0 acerca de cómo la sociología de la ciencia y la tecnología pueden beneficiarse mutuamente. En Thomas, H. y A. Buch, coord. Actos, actores y artefactos. Sociología de la tecnología. Bernal: Universidad Nacional de Quilmes Editorial. p. 19-62.

»Polino, Carmelo y Yurij Castelfranchi. 2012. The "communicate turn" in contemporary techno-science: Latin American approaches and global tendencies. En Schiele, B., M. Claessens y S. Sunke, eds. Science communication in the world: Practices, theories and trends. London: Springer.

»Price, Derek J. de Solla. 1965. The Science of Science. En Platt, J. T., ed. New views on the Nature of Man. Chicago: University of Chicago Press. p. 47-70.

» Rieger, Oya. 2008. Opening Up Institutional Repositories: Social Construction of Innovation in Scholarly Communication. En The Journal of Electronic Publishing. Vol. 11, no. 3. <https://doi.org/10.3998/3336451.0011.301>

» Rogers, Everett. 1962. Diffusion of innovations. New York: Macmillan.

"San Martín, Patricia, Paola Bongiovani, Ana Casali y Claudia Deco. 2013. Perspectivas socio-tecnológicas para el desarrollo de Repositorios de Acceso Abierto en el contexto de las universidades públicas de la región centro-este de Argentina. En IV Conferencia Internacional de la Public Knowledge Project. Universidad Nacional Autónoma de México.

»San Martín, Patricia, Griselda Guarnieri y Paola Bongiovani. 2014. Propuesta sociotecnológica para el desarrollo de repositorios de Acceso Abierto adecuados al contexto universitario argentino. En E-Ciencias de la Información. Vol. 4, no. 2, 2-27. <https://doi.org/10.15517/ECl.V4l2.15131>

»San Martín, Patricia, Guillermo Rodríguez, Marisa Cenacchi y Gonzalo Andrés. 2016. La construcción del espacio web de un instituto de investigación desde el abordaje socio-técnico. En Ciencia, Docencia y Tecnología. Vol. 27, no. 52, 402-422. <http://pcient.uner.edu.ar/cdyt/article/view/139> [Consulta: 17 enero 2020].

"Severin, Eugenio. 2013. Enfoques estratégicos sobre las TICs en Educación en América Latina y el Caribe. Santiago de Chile: UNESCO. <http://www.unesco.org/ new/fileadmin/MULTIMEDIA/FIELD/Santiago/images/ticsesp.pdf> [Consulta: 17 enero 2020]. 
» Tabares Quiroz, Juliana y Santiago Correa Vélez. 2014. Tecnología y sociedad: una aproximación a los estudios sociales de la tecnología. En Revista Ciencia, Tecnología y Sociedad. Vol. 9, no. 26, 129-144. <http://www.revistacts.net/ files/Volumen_9_Numero_26/Finales/TabaresFINAL.pdf> [Consulta: 17 enero 2020].

» Thomas, Hernán, Lucas Becerra y Agustín Bidinost. 2019. ¿Cómo funcionan las tecnologías? Alianzas socio-técnicas y procesos de construcción de funcionamiento en el análisis histórico. En Pasado Abierto. No. 10, 127-158. <http:// fh.mdp.edu.ar/revistas/index.php/pasadoabierto/article/view/3639> [Consulta: 17 enero 2020].

»Verón, Eliseo. 2015. Teoría de la mediatización: una perspectiva semio-antropológica. En CIC Cuadernos de Información y Comunicación. Vol. 20, 173-182. <https://doi.org/10.5209/rev_ciyc.2015.v20.50682> 
